\title{
Norovirus circulation in pediatric patients
}

Sara Giordana Rimoldi', Cristina Pagani', Fabrizio Stefani ${ }^{2}$, Nadia Zanchetta', Gian Vincenzo Zuccotti ${ }^{3}$, Ilaria di Bartolo4, Franco Ruggeri", Maria Rita Gismondo'

I Unità Operativa di Microbiologia e Virologia Azienda Ospedaliera e Polo Universitario Luigi Sacco

2 Dipartimento di Biotecnologie e Bioscienze, Università Bicocca Milano

3 Clinica pediatrica A.O. e Polo Universitario L.Sacco

4 Dipartimento di Sanità alimentare ed animale, Istituto Superiore di Sanità, Roma

Key words: Norovirus, sequencing, circulating strands, paediatric patients

Valutazione dei ceppi circolanti di Norovirus in pazienti pediatrici ricoverati per gastroenterite

\section{SUMMARY}

An eighteen-month study (2008-2009) was carried out on patients, aged on average 30 months, admitted with gastroenteritis to the Paediatric Unit of the L. Sacco Hospital in Milan, Italy. Faecal samples of I54 patients were investigated for Rotavirus, Norovirus, Adenovirus, Enterovirus and Bocavirus. A total of 25 norovirus positive samples were selected for nucleotide sequence analysis, that allowed identify GIl.2, GII.3 and Gll.4 2006 virus variants (06 a, 06 b).

\section{INTRODUZIONE}

Dal 2006 l’Unità Operativa Complessa di Microbiologia e Virologia dell’Azienda Ospedaliera Luigi Sacco in collaborazione con la Clinica Pediatrica dello stesso ente ha messo in atto una sorveglianza delle infezioni gastrointestinali in pazienti pediatrici ricoverati per gastroenterite acuta $(4,5)$; con il presente lavoro ci siamo prefissati di valutare l'epidemiologia dei ceppi circolanti di Norovirus mediante analisi di sequenza.

\section{METODI}

In un periodo compreso tra Gennaio 2008 e Ottobre 2009 sono stati collezionati 154 campioni fecali di pazienti pediatrici (età media 30 mesi, 80\% maschi) ricoverati per gastroenterite acuta, saggiati per Salmonella spp, Shigella spp, Clostridium difficile, Rotavirus, Norovirus, Bocavirus, Adenovirus, Enterovirus. I campioni risultati positivi alla real time PCR per Norovirus (Argene) sono stati sottoposti a RT/PCR impieganti due coppie di primers sulla regione capsidica ORF2 specifici per il genogruppo II (G2SKF/G2SKR) e I (G1SKF/G1SKR) di Norovirus umano e successiva PCR di sequenza. La comparazione delle sequenze nucleotidiche è stata eseguita con quelle depositate in banca dati (NCBI) (1).

\section{RISULTATI}

Venticinque campioni fecali risultati positivi per Norovirus in real time, sono stati saggiati con RT/PCR dando origine a una banda distinta di DNA in gel di agarosio della dimensione di circa 310 coppie di basi. L'analisi di sequenza, tuttavia, è stata possibile solo su 10 campioni.

La comparazione delle sequenze ottenute con quelle di riferimento nella banca dati ha rilevato un'identità nucleotidica del $100 \%$ confermando il coinvolgimento dello stesso ceppo virale.

In 6 dei 10 casi sottoposti a sequenziamento (60\%) è stata documentata la presenza del ceppo di Norovirus umano (Lordsdale) di genogruppo GII.4, variante 2006 b (GII.4 2006b), ceppo circolante in Europa durante il secondo semestre del 2006. Altri 2 ceppi, invece, hanno mostrato un'elevata omologia con il genogruppo GII.4 variante 2006a (20\%), che ha circolato nel primo semestre dello stesso anno. Due casi ulteriori si sono rivelati positivi per il ceppo GII.2 (10\%) e GII.3 (10\%) (Tabella 1).

\section{CONCLUSIONI}

I risultati ottenuti dall'analisi di sequenza di casi sporadici Norovirus collezionati in pazienti pediatrici affetti da gastroenterite acuta, ricoverati presso la Clinica Pediatrica durante 18 mesi di studio (2008-2009), provano un prevalente coinvolgimento del ceppo Lorsdale GII.4 varianti 2006b (60\%) e 2006 a (20\%) seguito dai genogruppi GII.2 (10\%) e GII.3 $(10 \%)$.

La circolazione dei tre differenti genotipi riportata nel nostro studio concorda con quanto documentato da altri autori $(2,3)$.

Tabella I. Risultati del sequenziamento dei ceppi di norovirus rilevati tra Gennaio 2008 e Ottobre 2009 in pazienti pediatrici ricoverati per gastroenterite acuta presso la Clinica Pediatrica dell'Azienda Ospedaliera L. Sacco.

\begin{tabular}{ccccc}
\hline Ceppi & Varianti & Età (mesi) & Sesso & casi \\
\hline & & I7 & & \\
GII.4 Lorsdale & \multirow{2}{*}{ 2006b } & 213 & & \\
& & 12 & $\mathrm{M}$ & 6 \\
& & 9 & & \\
\hline \multirow{2}{*}{ GII.4 Lorsdale } & \multirow{2}{*}{$2006 \mathrm{a}$} & $\mathrm{II}$ & & \\
\hline GII.2 & & 7 & $\mathrm{~F}$ & 2 \\
\hline GII.3 & & $\mathrm{II}$ & $\mathrm{M}$ & $\mathrm{I}$ \\
\hline
\end{tabular}

\section{BIBLIOGRAFIA}

1. Kojima S, Kageyama T, Fukushi S, et al. Genogroup-specific PCR primers for detection of Norwalk-like viruses. J Virol Methods 2002; 100: 107-14.

2. Medici MC, Martinelli M, Abelli LA, et al. Molecular epidemiology of norovirus infections in sporadic cases of viral gastroenteritis among children in northern Italy. J of Med Virol 2006; 78: 1486-92.

3. Medici MC, Martinelli M, Arcangeletti MC, et al. Epidemiological aspects of human rotavirus infection in children hospitalized with acute gastroenteritis in an area of northern of Italy. Acta Bio Medica 2004; 75: 100-6.

4. Rimoldi SG, Bartolone R, Calvagna N, et al. New viral gastrointestinal agents associated with paediatrics illness. Microbiologia Medica 2009; 24 (2): $92-5$

5. Rimoldi SG, Pagani C, Lombardi A, et al. Epidemiological evaluation of sporadic cases of Norovirus infection in communitary and hospitalized patients. Microbiologia Medica 2009; 24 (1): 47-9. 